\title{
STATISTICAL ASSESSMENT OF FATIGUE-INITIATING MICROSTRUCTURAL FEATURES IN A POLYCRYSTALLINE DISK ALLOY
}

\author{
William C. Lenthe ${ }^{1}$, Jean-Charles Stinville ${ }^{1}$, McLean P. Echlin ${ }^{1}$, Tresa M. Pollock ${ }^{1}$ \\ ${ }^{1}$ Materials Department; University of California, Santa Barbara; Santa Barbara, CA 93106
}

Keywords: Fatigue, Twin Boundary, Serial Sectioning, Property Volume Element, René 88DT

\begin{abstract}
Fatigue is the life limiting property of polycrystalline nickel-base superalloys used for turbine disks. Fatigue cracks in René 88DT frequently initiate in large grains that are in the tail of the size distribution and contain favorably oriented annealing twin boundaries. High resolution three dimensional volumes of René 88DT have been collected via TriBeam tomography for analysis of grain structure and twin boundaries. Most twin boundaries were found to be highly coherent, allowing twin boundary inclination to be calculated using orientation measurements collected from two dimensional cross sections. This insight enables identification of fatigue initiating microstructural features in large two dimensional scans, providing initiation site statistics for a significant number of boundaries.
\end{abstract}

\section{Introduction}

Fatigue is the life limiting property of polycrystalline nickel-base superalloys used widely in turbine disks for power generation and aerospace applications. While fatigue cracks may initiate at large pores or inclusions, such extrinsic defects are uncommon in alloys processed through advanced powder metallurgical (PM) routes. In the absence of such large imperfections, initiation occurs at intrinsic defects making fatigue life highly microstructure sensitive and lifetime prediction challenging $[1,2]$. In high cycle fatigue, crack formation and initial growth can account for $80 \%$ of fatigue life and the initiation processes results in a large fatigue life scatter [3, 4]. Identifying and characterizing regions amenable to crack nucleation and initial propagation is a critical step in both predicting fatigue life of current materials and designing new materials with improved fatigue life.

Polycrystalline superalloys in the high or very high cycle fatigue regime can have lifetimes exceeding $10^{9}$ cycles and may not exhibit endurance limits $[5,6]$. As a result a single fatigue test requires months or years using traditional methods. Ultrasonic fatigue testing at a frequency of $20 \mathrm{kHz}$ makes it possible to apply $10^{9}$ cycles in a single day, greatly increasing experiment throughput. Recent efforts also enable in-situ damage detection allowing precise interruption between crack initiation and failure. Coupled with the ability to perform testing at elevated temperatures $[6,7]$, these developments have provided new insights to the microstructural features responsible for crack nucleation in polycrystalline-nickel-base superalloys $[8,9]$.

Fatigue cracks in the PM disk alloy René 88DT initiate due to strain localization near annealing twin boundaries in large grains that are favorably oriented for slip (possessing a high Schmid factor). Accumulation of irreversible, cyclic dislocation slip results in large incompatibility stresses, ultimately nucleating a crack [8].

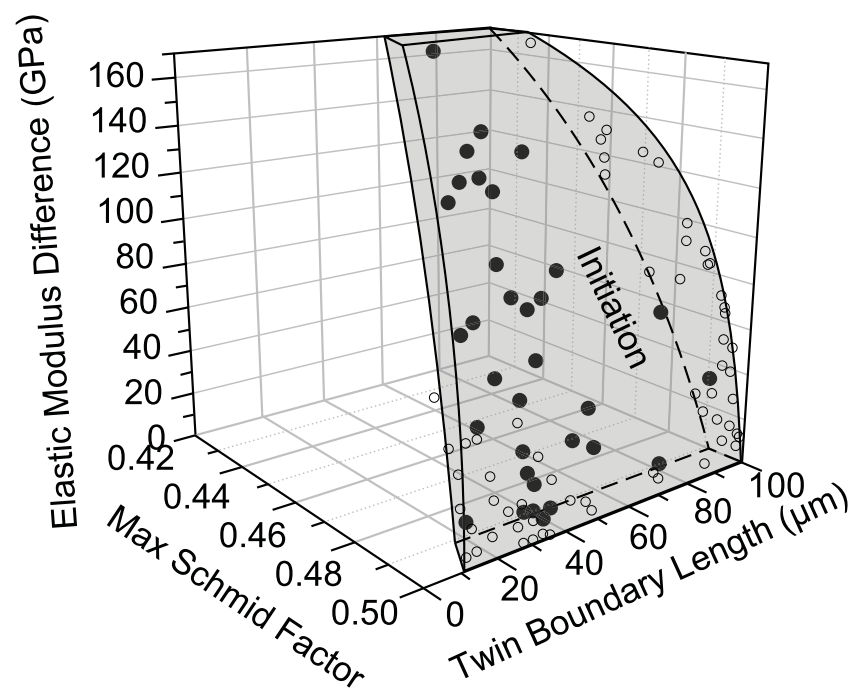

Figure 1. The fatigue crack initiation criterion in René 88DT can be defined by modulus mismatch, boundary length, and maximum Schmid factor for coherent twin boundaries with the active slip system parallel to the coherent plane (after Stinville et al. [4]).

Surface investigations of interrupted fatigue tests for René 88DT similarly found that cracks initiate near long twin boundaries in favorably orientated grains with the highest Schmid factor slip system trace parallel to the twin boundary [4]. Based on these observations, a multi-parameter criterion for crack initiating twin boundaries, shown in Figure 1, has recently been developed [4]. Twin boundaries have also been observed to play a role in crack initiation in a variety of other FCC material systems including stainless steel and copper $[10,11]$.

When considering the mean value of a microstructural feature or a property, along with statistical deviation from the mean value, a proper sampling framework is critical [12]. The relevant feature or property is repeatedly measured with samples of varying size. Volumes small with respect to the relevant characteristic length scale will have high variability with scatter decreasing at larger volumes. If the convergence behavior is well characterized, scatter can be predicted as a function of interrogated volume [12].

In general, two dimensional planar boundaries, including twin and grain boundaries can be mathematically described by five parameters. Two are needed to define the boundary plane orientation and an additional three are needed to define the misorientation across the boundary [13]. These boundaries cannot be fully characterized from a two dimensional cross section since the boundary inclination is unknown. Historically, collecting three 
dimensional microstructure volumes has been time consuming and required large amounts of human intervention [14,15]. Presently, moderate volumes of $3 \mathrm{D}$ microstructure data can be collected semi or fully automatically using mechanical polishing or a focused ion beam (FIB) in a scanning electron microscope (SEM) to remove thin layers of material $[16,17]$. Crystallographic orientation can be mapped using electron backscatter diffraction (EBSD), however slow FIB material removal rates restrict accessible volumes and achieving consistent removal rates with mechanical polishing is challenging. Recently, a femtoseccond laser has been coupled with a dual beam FIB-SEM allowing comparable in plane resolution with material removal rates 4-5 orders of magnitude faster than a FIB while introducing minimal damage $[18,19]$. This TriBeam system enables rapid 3D characterization of a wide variety of material systems $[20,21]$.

Although twin boundaries are crucial microstructural features that influence fatigue crack initiation in René 88DT (and likely many other nickel-based alloys) their 3D structure has not been thoroughly studied. In this research, high resolution 3D volumes of René 88DT have been collected via TriBeam tomography and twin boundary coherence characterized. Based on the finding that twin boundaries are highly coherent in three dimensions, we show that crack initiation sites can be rigorously identified in $2 \mathrm{D}$ datasets, enabling measurement of fatigue initiation site frequency for statistically significant numbers of grains.

\section{Methodology}

EBSD datasets including 3D volumes and $\mathrm{mm}^{2}$ scale $2 \mathrm{D}$ cross sections were collected from René 88DT fabricated through a powder metallurgy route. The forged and heat treated material has no significant crystallographic texture and an average grain size of $26 \mu \mathrm{m}$. The nominal composition is given in Table I [22]. René 88DT has a bimodal distribution of $\gamma^{\prime}$ precipitates with 100-200 $\mathrm{nm}$ secondary and $\sim 10 \mathrm{~nm}$ tertiary precipitates [23].

Table I. Composition of René 88DT in Weight Percent

\begin{tabular}{ccccccccccc}
\hline $\mathrm{Ni}$ & $\mathrm{Co}$ & $\mathrm{Cr}$ & $\mathrm{Mo}$ & $\mathrm{W}$ & $\mathrm{Al}$ & $\mathrm{Ti}$ & $\mathrm{Nb}$ & $\mathrm{C}$ & $\mathrm{B}$ & $\mathrm{Zr}$ \\
\hline bal. & 13 & 16 & 4 & 4 & 2.1 & 3.7 & 0.7 & 0.03 & 0.015 & 0.03 \\
\hline
\end{tabular}

\section{D Characterization}

Data Collection A $10 \times 5 \times 1 \mathrm{~mm}$ sample was sectioned from a plate of René 88DT using wire electrical discharge machining (EDM). A series of $600 \mu \mathrm{m}$ wide pedestals were fabricated with wire EDM and then mechanically polished to $600 \mu \mathrm{m}$ creating a series of $600 \times 600 \mu \mathrm{m}$ pedestals as shown in Figure 2. A single pedestal was destructively sectioned via femtosecond laser ablation in the TriBeam system. The $780 \mathrm{~nm}$ laser was attenuated to $40 \mathrm{~mW}$ and scanned parallel to the sample surface, raising the sample $250 \mathrm{~nm}$ into the beam each slice. After each cut a 100 $\mathrm{nm}$ wide region was cleaned with a $15 \mathrm{nA} \mathrm{Ga}^{+}$FIB with a 30 $\mathrm{kV}$ accelerating voltage at a glancing angle of $3^{\circ}$. FIB cleaning improves EBSD pattern quality by removing a thin layer of high dislocation density material and reducing surface roughness due to laser induced periodic surface structures [19]. EBSD was collected from a $75 \mathrm{x} 100 \mu \mathrm{m}$ area within the cleaned region using a $25 \mathrm{kV}$ electron beam, $8 \times 8$ camera binning, and a $100 \mathrm{~nm}$ step size. A total

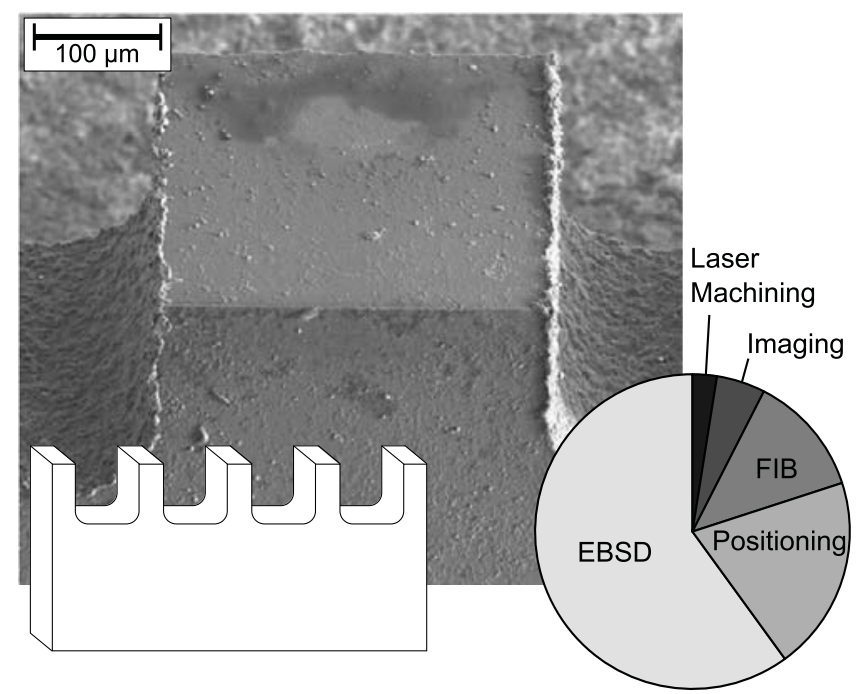

Figure 2. A series of $600 \mu \mathrm{m}$ wide pedestals was fabricated via wire EDM from a 10 × 5 x $1 \mathrm{~mm}$ strip (bottom left). The strip was mechanically polished to $600 \mu \mathrm{m}$ creating $600 \times 600 \mu \mathrm{m}$ pedestals. Each slice took 40 minutes to collect with the time distribution per slice shown in the pie chart (bottom right).

volume of $75 \times 100 \times 104 \mu \mathrm{m}$ was collected from 139 ablated slices. The cut pedestal and FIB cleaned region are shown in Figure 3 with the area of EBSD acquisition outlined. Collection of each slice required 40 minutes of instrument time with 24 minutes of EBSD accounting for the majority.

Data Reconstruction Reconstruction and analysis of EBSD data was performed using the DREAM.3D software package [24]. Rotations of $180^{\circ}$ about [010] and $90^{\circ}$ about the [001] were applied to the sample and orientation reference frames respectively

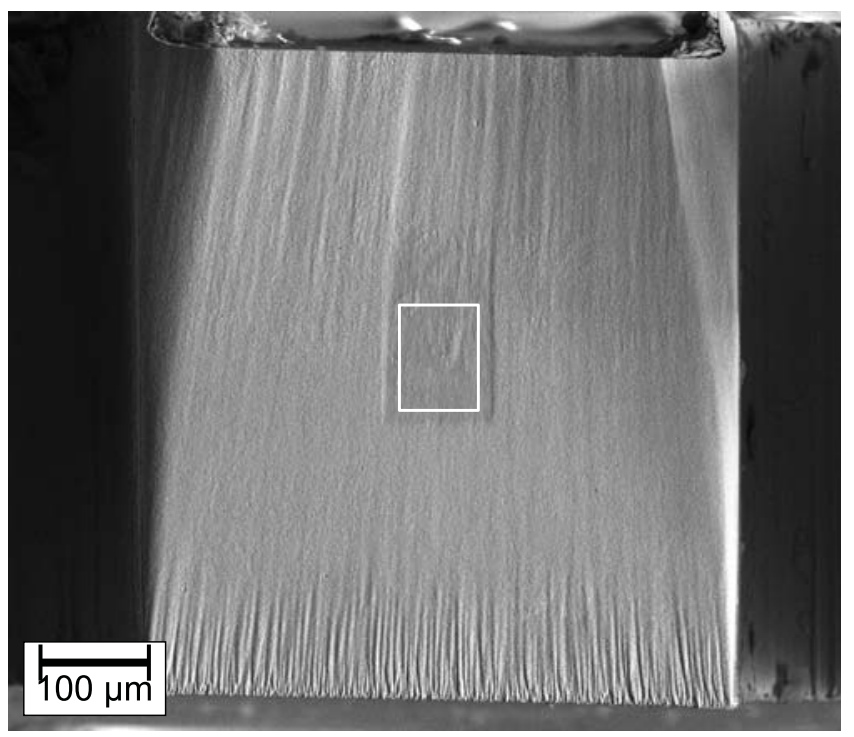

Figure 3. A secondary electron image of the sample pedestal is shown after laser cutting and FIB cleaning. The EBSD scan area is outlined in white. 

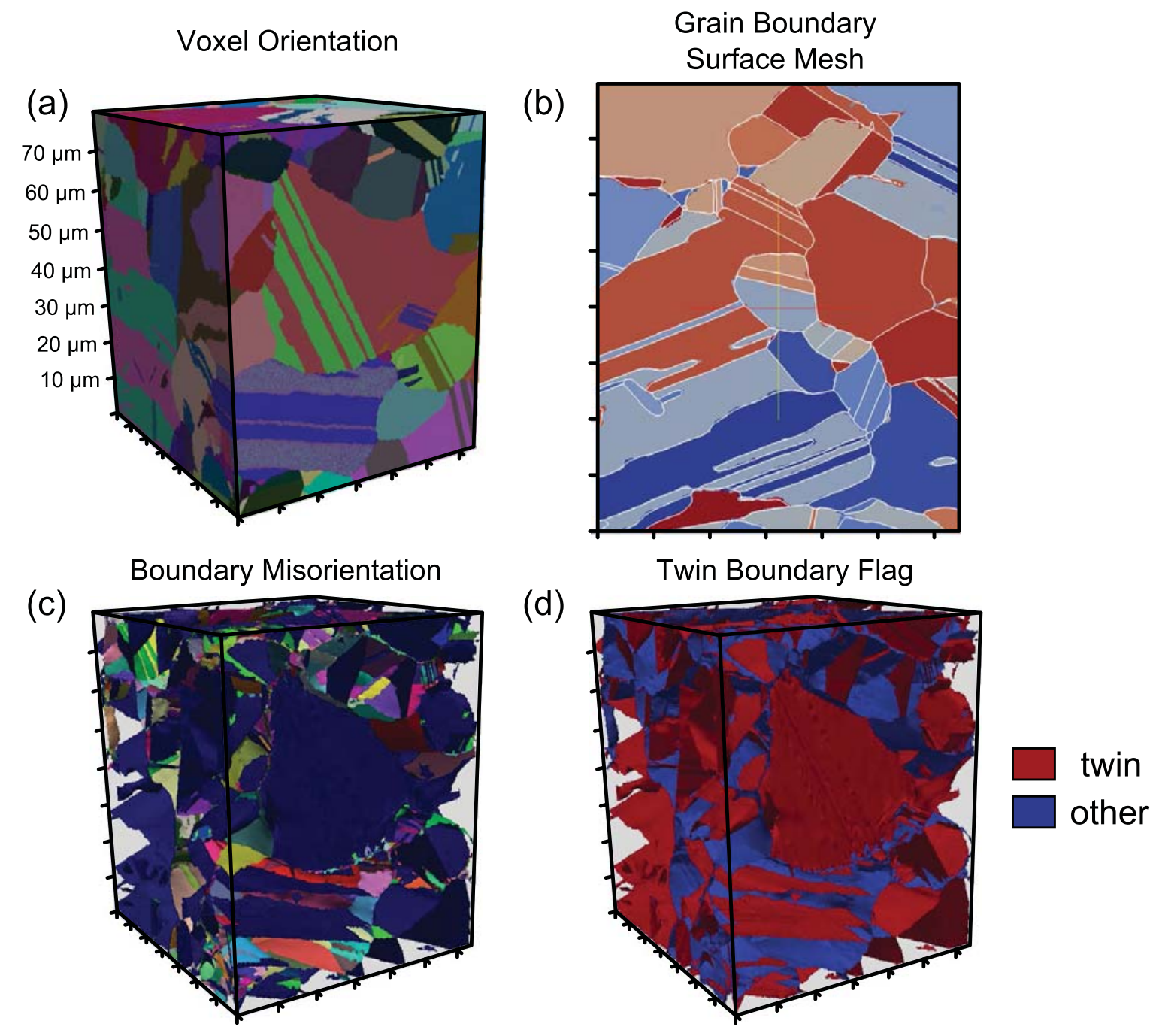

Twin Coherence

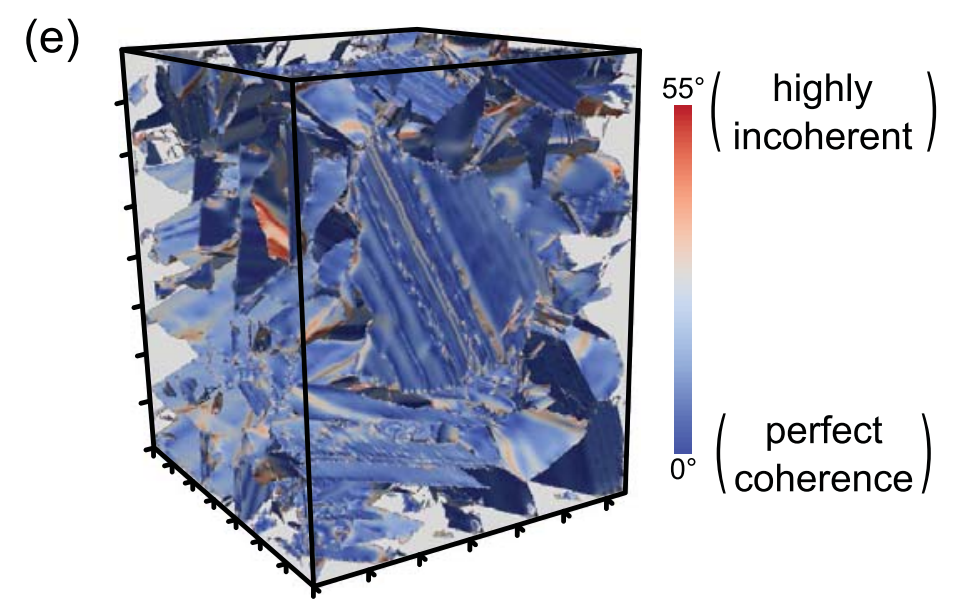

Figure 4. A summary of the 3D dataset reconstruction and surface mesh analysis is presented. (a) The aligned dataset is colored by orientation. (b) Grains defined with a $2^{\circ}$ segmentation tolerance are meshed with a multi-material marching cubes algorithm and Laplacian smoothed (representative cross section). (c) The misorientation across each face is computed. (d) Twin boundaries (red) are identified with a tolerance on misorientation. (e) Incoherence angle is computed for each face. The electronic version of this article has color images. 


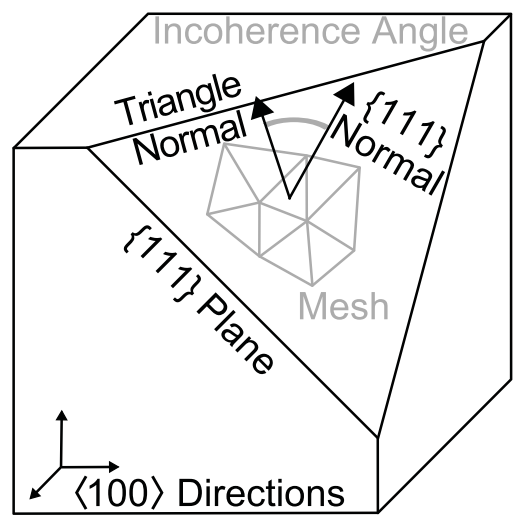

Figure 5. The calculation of incoherence angle for a twin boundary face and a single bounding orientation is shown schematically.

to account for manufacturer definitions. A threshold of 0.05 on confidence index (CI) and 1000 on image quality (IQ) was applied to remove any poorly indexed voxels. Next slices were stacked and aligned by maximizing voxels falling within a $5^{\circ}$ disorientation angle tolerance relative to the previous slice. After computing the aligned slice positions a linear regression was performed and the resulting linear background subtracted to prevent shearing of the dataset due to a nonzero average shift. Grains were defined in the aligned dataset using a $2^{\circ}$ segmentation tolerance. Grains smaller than 25 voxels (an equivalent diameter of $0.62 \mu \mathrm{m}$ ) were removed by isotropically dilating their neighbors.

Following reconstruction, a grain boundary surface mesh was generated using a multi-material marching cube algorithm [25]. Laplacian smoothing was applied to the resulting mesh to remove aliasing artifacts [26]. The resulting surface mesh closely follows grain boundaries in the voxelized dataset with minimal artificial roughness as seen in Figure 4(b). The misorientation across each triangle was computed using the average orientations of the two bounding grains. Triangles with a misorientation angle within $5^{\circ}$ of $60^{\circ}$ and a misorientation axis within $5^{\circ}$ of $\{111\}$ are defined as twin boundaries. An incoherence angle is calculated for each twin boundary face by measuring the angle between the triangle normal and the shared $\langle 111\rangle$ direction as shown in Figure 5. The incoherence angle is measured with respect to both bounding orientations and averaged. The full process for computing twin boundary coherence is shown schematically in Figure 4 with a misorientation coloring legend available in Figure 6.

\section{D Dataset}

Data Collection A sample was cut from a plate of René 88DT and metallographically prepared with silicon carbide papers. The sample was vibratory polished in colloidal silica for 12 hours and a $1.0 \times 1.6 \mathrm{~mm}$ EBSD scan collected using a $20 \mathrm{kV} 0.2 \mathrm{nA}$ electron beam with a $800 \mathrm{~nm}$ step size and $4 \times 4$ camera binning.

Data Reconstruction A threshold of 0.1 on confidence index (CI) and 700 on image quality (IQ) was applied to remove any poorly indexed voxels [28]. Grains were defined using a $2^{\circ}$ segmentation tolerance and grains smaller than 9 voxels (an equivalent diameter of $2.7 \mu \mathrm{m}$ ) removed by isotropically dilating their neighbors. For
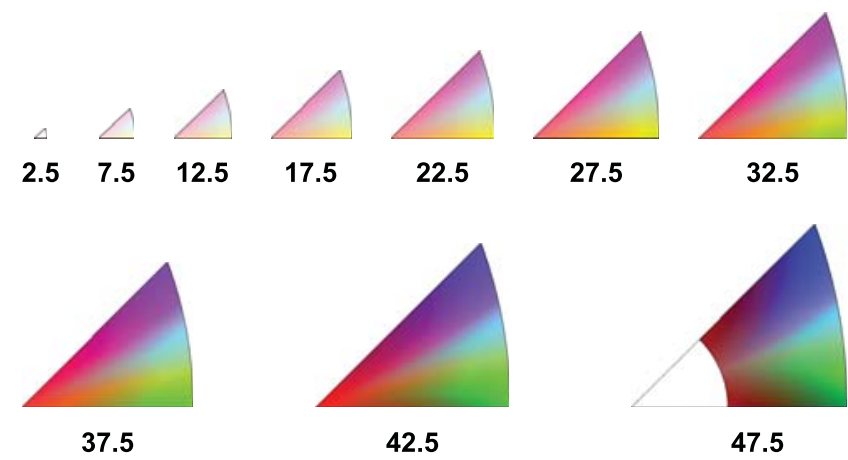

22.5

27.5

32.5
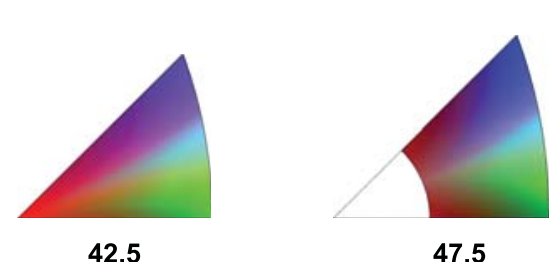

47.5
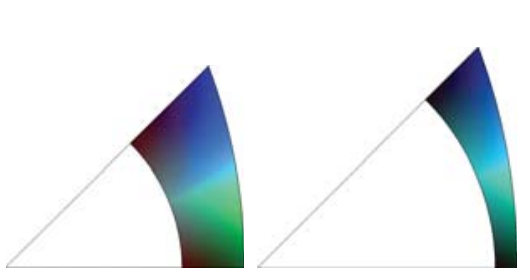

57.5

$[001]$

[111]

Figure 6. A misorientation coloring legend is shown [27]. To map a misorientation to a color first select the stereographic triangle corresponding to the misorientation angle, then identify the color using the misorientation axis as with an inverse pole figure color mapping.

each grain the Schmid factor for [010] loading and the elastic modulus in the [010] direction were computed using the grain average orientation.

A grain boundary surface mesh was generated and twin boundary and line segments classified using a $5^{\circ}$ tolerance on misorientation axis and misorientation angle. To compute the coherent direction of each twin boundary in the sample reference frame, all four $\{111\}$ directions of the first bounding grain are rotated to the crystallographic reference frame of the second bounding grain. Each rotated direction is compared to the $\{111\}$ directions of the second bounding grain and the most parallel pair determined. The pair is averaged and the resulting coherent direction rotated to the sample reference frame. Using the computed coherent direction, twin boundaries parallel to highest Schmid factor slip systems of their bounding grains can be identified. The boundary area is computed without mesh smoothing by projecting segments onto the coherent twin trace. Likely fatigue initiating twin boundaries were identified with a threshold on modulus mismatch, boundary length, and Schmid factor for all boundaries with the highest Schmid factor slip system parallel to the coherent twin plane [4].

The dataset was subsampled to investigate the microstructure volume element size relevant to fatigue crack initiation [12]. Square volume elements with edge lengths ranging from 5-500 $\mu \mathrm{m}$ were used to calculate the frequency of initiation sites. At each size, 50 volumes were randomly sampled from the full dataset. The length of all mesh segments falling completely within the volume element were accumulated and the line fraction of boundaries satisfying the initiation criterion measured (Figure 7). 


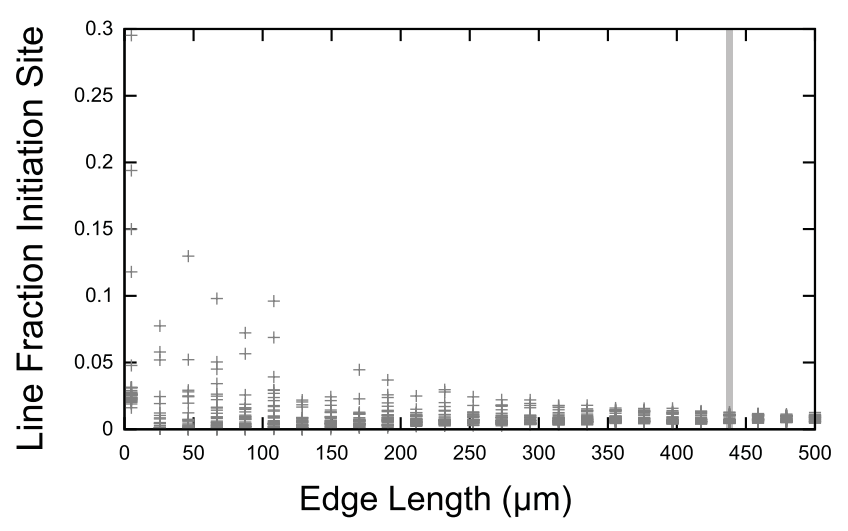

Figure 7. The line fraction of fatigue crack initiating boundaries plotted against sampling box size with 15 boxes selected at random for each size. The vertical line indicates $95 \%$ confidence convergence to within $5 \%$ of the sample mean.

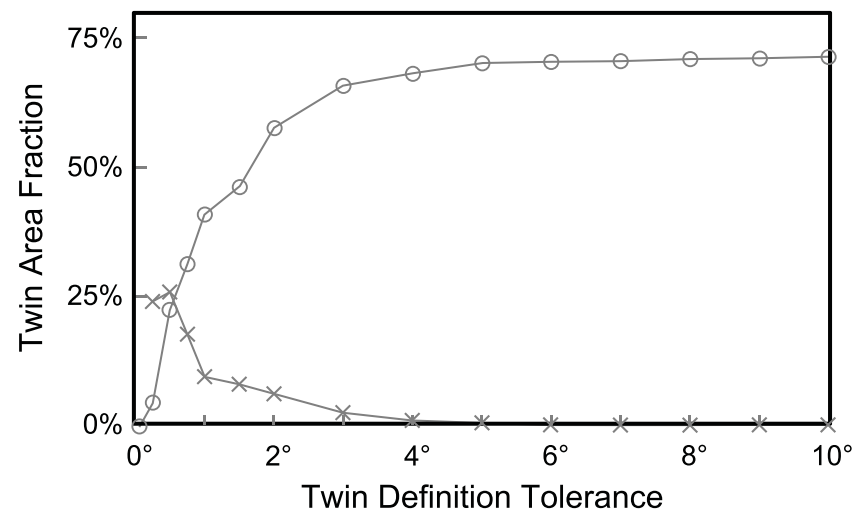

Figure 8. Area fraction twin boundary plotted against tolerance of twin definition. Circles and crosses indicate cumulative and additional area fraction respectively.

\section{Results and Discussion}

Twin boundaries in René 88DT have been quantitatively characterized in three dimensions. A range of tolerances for identifying twin boundaries was applied as shown in Figure 8, revealing that a $5^{\circ}$ cutoff for both misorientation axis and misorientation angle provides a suitable balance between excluding twin boundaries deviating from exactly $60^{\circ}$ about $\{111\}$ and including random boundaries close to a twin misorientation. The twin boundary area fraction asymptotically approaches $70 \%$ as the tolerance is relaxed exhibiting a high sensitivity below $3^{\circ}$ and a relatively flat response between $5^{\circ}$ and $10^{\circ}$. At higher thresholds significant percentages of random misorientations begin to be captured. The high degree of twinning creates an incredibly complex boundary network as shown in Figure 9 making a single misorientation insufficient to describe boundaries between grains with twins removed.

An area weighted histogram for twin incoherence angle is shown in Figure 10, revealing that most twins are highly coherent; $80 \%$ of twin boundary area has an incoherence angle less than $20^{\circ}$ and $95 \%$ has an incoherence angle less than $30^{\circ}$. Artificial roughness
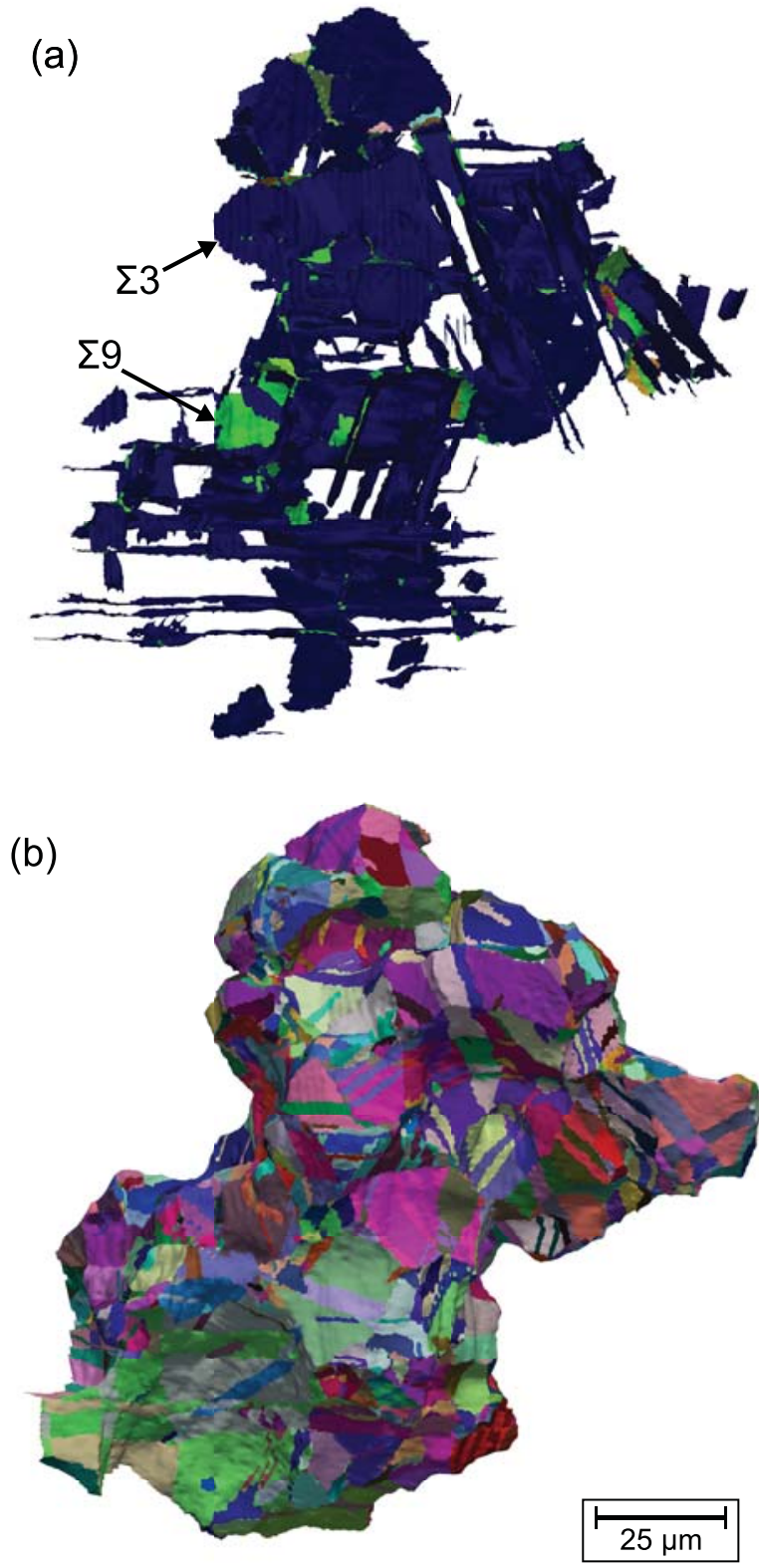

Figure 9. (a) The internal boundaries for a single large reconstructed grain are shown with dark blue and green corresponding to $\Sigma 3$ and $\Sigma 9$ boundaries respectively. (b) The complex internal twin structure of each individual grain compounds for boundaries with neighboring grains resulting in a myriad of misorientations.

introduced to the surface mesh due to the voxelized input caused the mesh faces to deviate from perfect coherence. Laplacian smoothing significantly reduces this artifact but long range, low amplitude ripples persist, adding a finite value to the incoherence angle. Sharp steps have been observed experimentally on twin boundaries in René 88DT [8] and twins frequently terminate within a grain geometrically requiring regions of incoherent twin boundary area. 


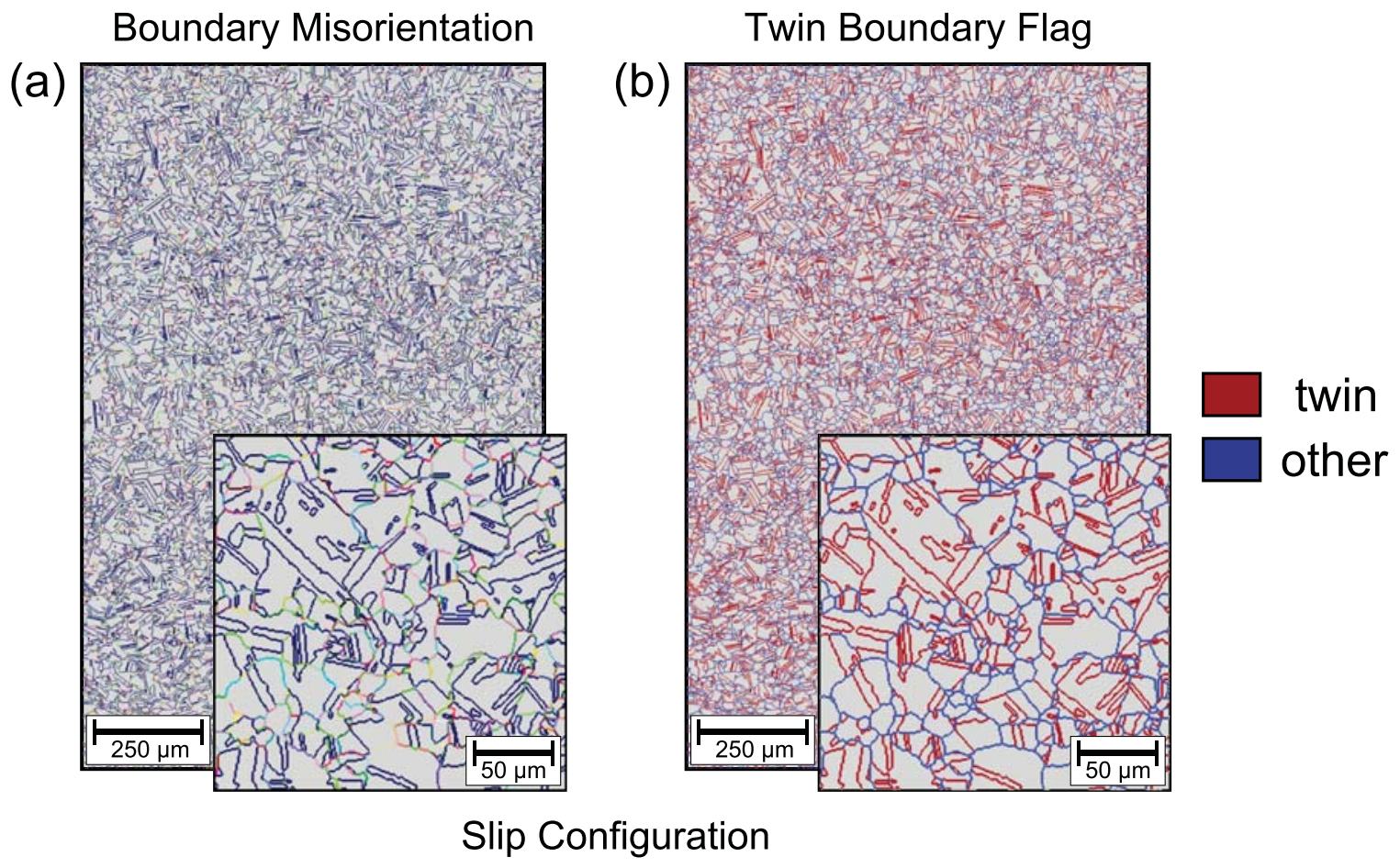

(c)

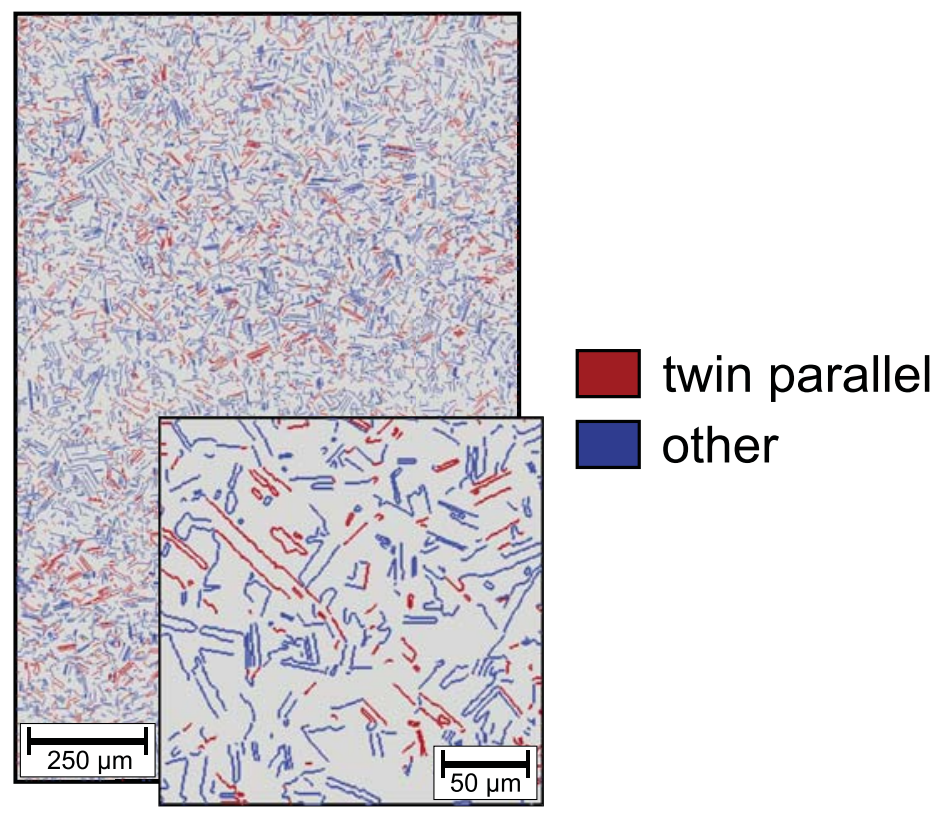

Figure 11. A summary of the 2D dataset reconstruction and surface mesh analysis is presented. (a) Grains defined with a $2^{\circ}$ segmentation tolerance are meshed and the misorientation across each face is computed. (b) Twin boundaries (red) are identified with a tolerance on misorientation. (c) The coherent direction for each twin boundary is computed and boundaries with the highest Schmid factor slip system parallel to the coherent plane identified. 
Boundary Misorientation

(a)

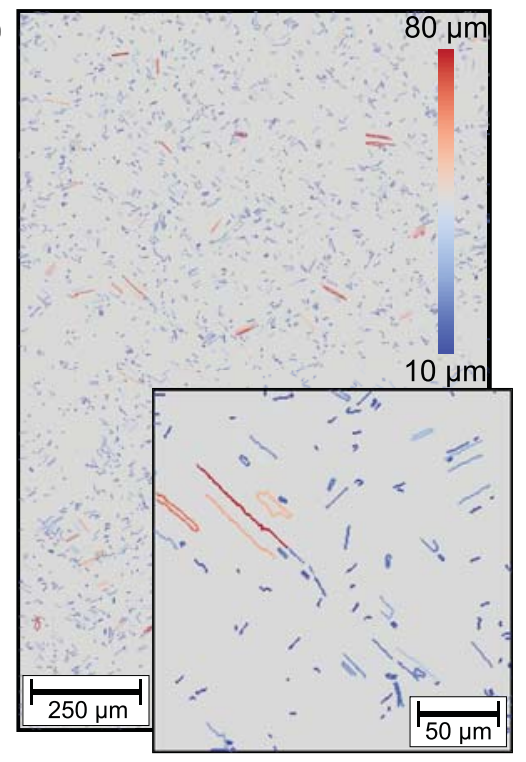

Schmid Factor

(c)

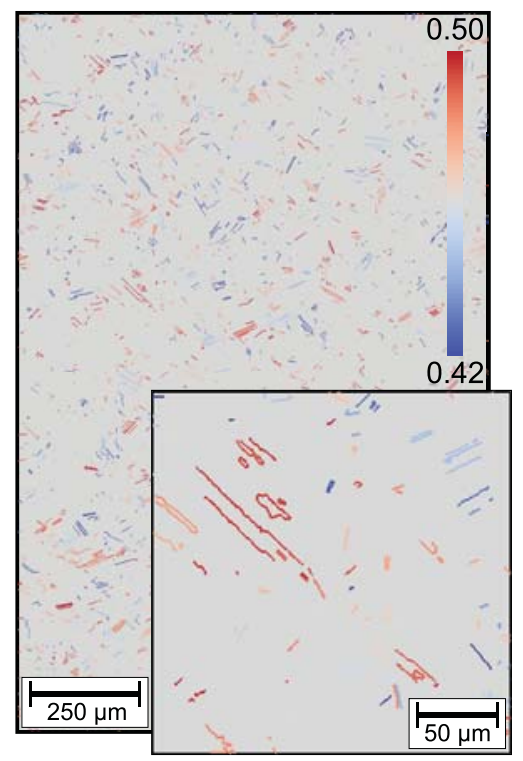

Twin Boundary Flag

(b)

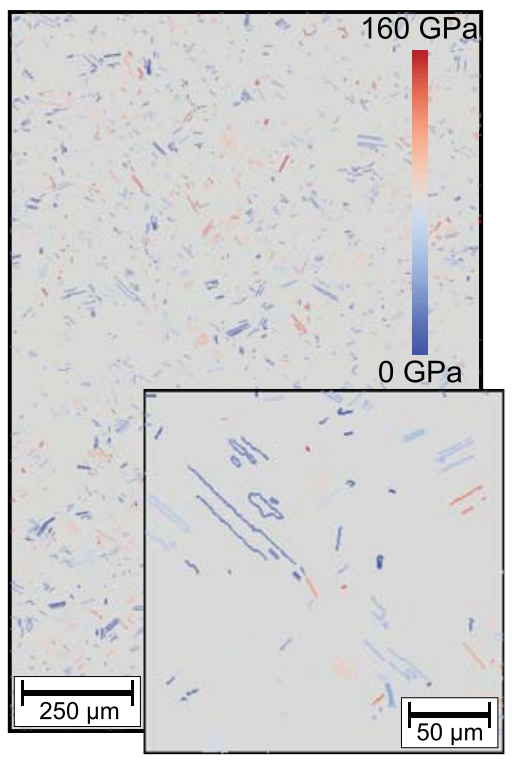

Initiation Flag

(d)

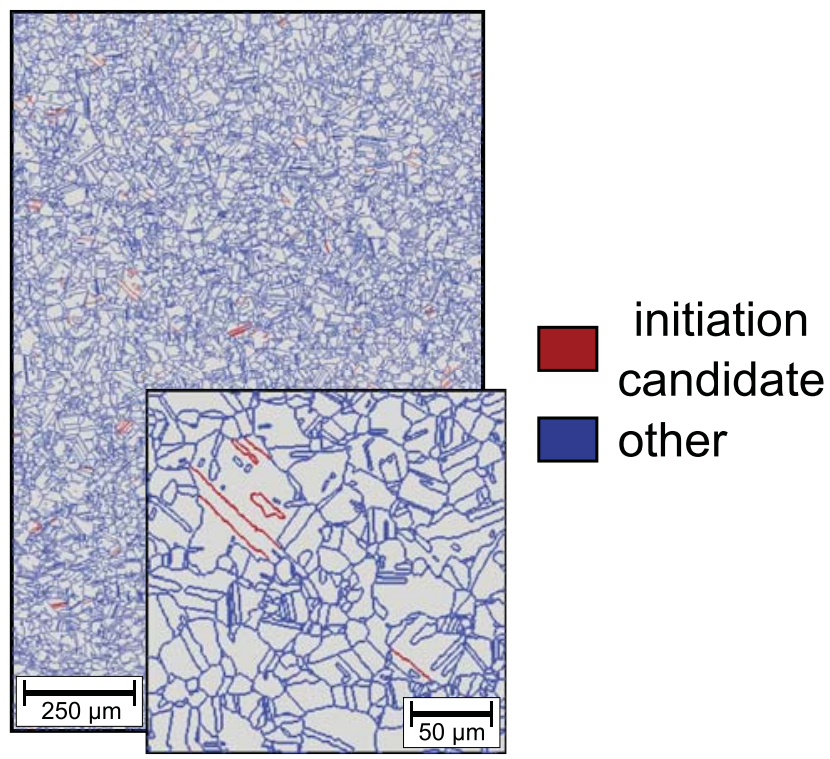

Figure 12. A summary of the 2D dataset reconstruction and surface mesh analysis is presented. (a) the length for each boundary is computed by projection along the coherent plane trace. (b) the elastic modulus mismatch is computed for each boundary. (c) the maximum Schmid factor for each grain is computed. (d) The criterion for fatigue crack initiation is applied using a threshold on twin boundary length, elastic modulus mismatch, and twin boundary parallel Schmid factor and crack initiation sites are identified (red). Boundaries meeting the initiation criterion are often clustered as in the large grain in the upper left of the inset in image (d) which contains 5 candidate boundaries. 


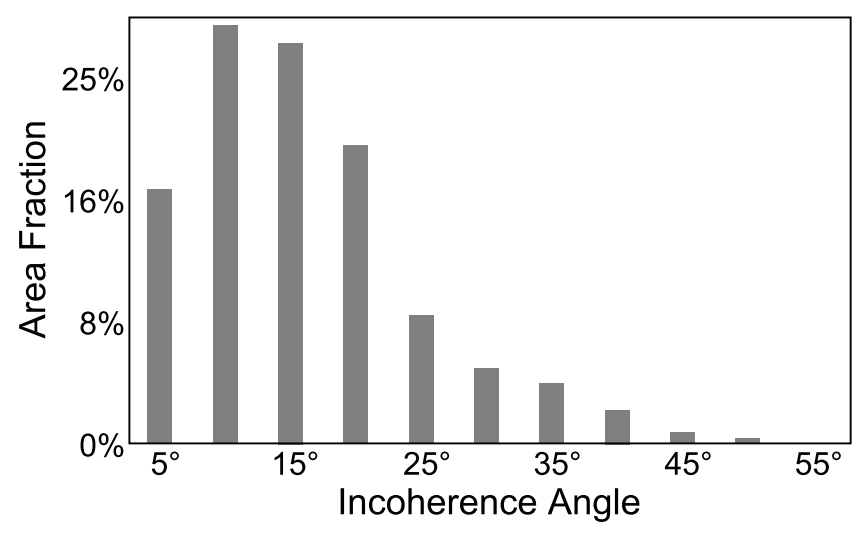

Figure 10. An area weighted twin coherence histogram is plotted.

Approximately $75 \%$ of all twin boundary area is coherent (Figure 10). By assuming coherence, the inclination of each twin boundary can be calculated allowing identification of fatigue crack initiation sites in a 2D section as shown in Figure 11 and Figure 12. The $1.0 \times 1.6 \mathrm{~mm}$ EBSD scan contained over 65,000 grain boundaries of which only 146 satisfy the criterion for crack initiation. The number density of 90 crack initiation candidates per $\mathrm{mm}^{2}$ is roughly an order of magnitude greater than experimentally observed crack densities of 10 per $\mathrm{mm}^{2}$ [4]. However, twin boundaries that meet the initiation criterion are frequently clustered as shown in Figure 12 artificially increasing the density of crack initiation candidates.

A property volume element (PVE) for fatigue crack initiation was defined by sampling areas from the scan with a range of sizes (Figure 7). The least squares power law curve fit for standard deviation of fatigue crack initiating boundary fraction is $1.15 *$ area $^{-0.5}$. Convergence, defined as a $95 \%$ confidence that the population mean is within $10 \%$ of the sample average occurs for areas greater than $440 \times 440 \mu \mathrm{m}$. 3D measurement of initiation candidate density would correspondingly require collecting the volume beneath an area of $440 \times 440 \mu \mathrm{m}$, enabling consideration of the stress state at each boundary by simulated loading. If a more stringent criterion is enforced, e.g. a 99\% confidence that the population mean is within $5 \%$ of the sample mean, convergence does not occur for the entire dataset $1.0 \times 1.6 \mathrm{~mm}$ dataset, suggesting the property volume element is larger than the area sampled.

Since small crack growth can also account for a significant fraction of the total fatigue life, a propagation criterion is required to determine the PVE for fatal cracks. Although simple misorientation criteria exist [9], they are unsuitable for René 88DT given the complex grain boundaries resulting from high twin density as shown in Figure 9. A propagation criterion is currently being developed which will allow measurement of the PVE for fatal crack density, shown schematically in Figure 13, with the 0.2 $\mathrm{mm}^{2}$ fatigue crack initiation density PVE providing a lower bound. Interrogating the fatal crack density PVE for René 88DT will permit prediction of fatigue life variability. Using this framework, it is possible to determine fatigue crack initiation density for synthetic microstructure instantiations of powder metallurgy processed polycrystalline alloys. Parameter studies on

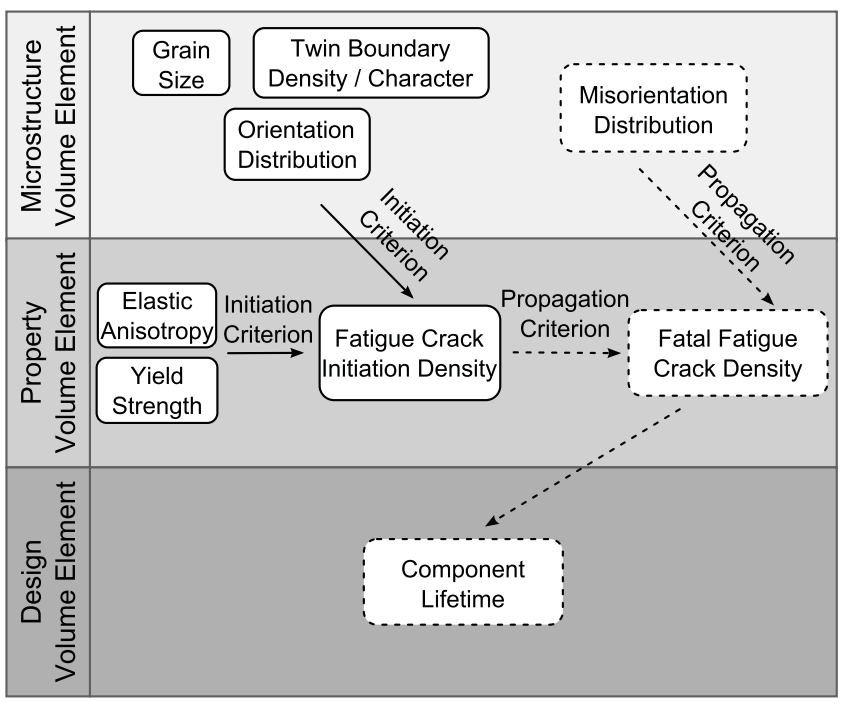

Figure 13. The average incidence of initiation sites and variability of fatigue crack initiation density is dependent on the values and variabilities of contributing microstructures and properties through the initiation criterion. A fatal crack density PVE will be determined in turn by implementing a propagation criterion. Variability in fatal crack density ultimately guides design and predicting component lifetimes.

the influence of twin density and other microstructural parameters can aid in development of materials with improved fatigue life.

\section{Conclusions}

1. $70 \%$ of boundary area in René $88 \mathrm{DT}$ is twin boundary when measured in 3D.

2. $80 \%$ of twin boundary area in René 88DT has an incoherence angle less than $20^{\circ}$.

3. Fewer than $0.25 \%$ of grain boundaries in René 88DT satisfy the criteria for nearby crack initiation.

4. The property volume element for fatigue crack initiation density in René 88DT is at least $0.2 \mathrm{~mm}^{2}$ when initiation occurs at long twin boundaries with high elastic modulus mismatch and a large resolved shear stress on the slip system parallel to the boundary.

This work has been supported through the AFOSR/AFRL sponsored Center of Excellence on Integrated Materials Modeling (CEIMM) grant No. FA9550-12-1-0445 and GE Global Research. The authors gratefully acknowledge Micheal Groeber and Micheal Jackson for DREAM.3D development support and Micheal Uchich and Micheal Groeber for useful discussions.

\section{References}

1. T.M. Pollock and S. Tin, "Nickel-Based Superalloys for Advanced Turbine Engines: Chemistry, Microstructure and Properties," Journal of Propulsion and Power, 22 (2) (2006), 361-374. 
2. B.A. Cowles, "High Cycle Fatigue in Aircraft Gas Turbines-an Industry Perspective," International Journal of Fracture, 80 (2-3) (1989), 147-163.

3. A. Kumar et al., "In Situ Characterization of Fatigue Damage Evolution in a Cast Al Alloy Via Nonlinear Ultrasonic Measurements," Acta Materialia, 58 (6) (2010), 2143-2154.

4. J.C. Stinville et al., "A Combined Grain Scale Elastic-Plastic Criterion for Identification of Fatigue Crack Initiation Sites in a Twin Containing Polycrystalline Nickel-Base Superalloy," Acta Materialia, 103 (2016), 461-473.

5. C. Bathias, "There is No Infinite Fatigue Life in Metallic Materials," Fatigue \& Fracture of Engineering Materials \& Structures, 22 (7) (1999), 559-565.

6. A. Shyam et al.. "Development of Ultrasonic Fatigue for Rapid, High Temperature Fatigue Studies in Turbine Engine Materials," Superalloys 2004, ed. K.A. Green et al. (Warrendale, PA: The Minerals, Metals \& Materials Society, 2004), 19-23.

7. J.Z. Yi et al., "Ultrasonic Fatigue of a Single Crystal Ni-Base Superalloy at 1000 C," Materials Science and Engineering: A, 443 (1) (2007), 142-149.

8. J. Miao, T.M. Pollock, and J.W. Jones, "Crystallographic Fatigue Crack Initiation in Nickel-Based Superalloy René 88DT at Elevated Temperature," Acta Materialia, 57 (20) (2009), 5964-5974.

9. J. Miao, T.M. Pollock, and J.W. Jones, "Microstructural Extremes and the Transition From Fatigue Crack Initiation to Small Crack Growth in a Polycrystalline Nickel-Base Superalloy," Acta Materialia, 60 (6) (2012), 2840-2854.

10. M. Mineur, P. Villechaise, and J. Mendez, "Influence of the Crystalline Texture on the Fatigue Behavior of a 316L Austenitic Stainless Steel," Materials Science and Engineering: A, 286 (2) (2000), 257-268.

11. R.C. Boettner, A.J. McEvily Jr, and Y.C. Liu, "On the Formation of Fatigue Cracks at Twin Boundaries," Philosophical Magazine, 10 (103) (1964), 95-106.

12. M.P. Echlin, W.C. Lenthe, and T.M. Pollock, "Three-Dimensional Sampling of Material Structure for Property Modeling and Design," Integrating Materials and Manufacturing Innovation, 3 (1) (2014).

13. A. Morawiec and K.L. Merkle. "Symmetries of Grain Boundary Distributions," Proceedings of the Third International Conference on Grain Growth, ed. H. Weiland, B.L. Adams, and A.D. Rollet (Warrendale, PA: The Minerals, Metals \& Materials Society, 1998), 509.

14. M.V. Kral and G. Spanos, "Three-Dimensional Analysis of Proeutectoid Cementite Precipitates," Acta Materialia, 47 (2) (1999), 711-724.

15. M.V. Kral et al., "Three-Dimensional Analysis of Microstructures," Materials Characterization, 45 (1) (2000), $17-23$.
16. A.D. Rollett et al., "Three-Dimensional Characterization of Microstructure by Electron Back-Scatter Diffraction," Annual Review of Materials Research, 37 (2007), 627-658.

17. M.D. Uchic, M.A. Groeber, and A.D. Rollett, "Automated Serial Sectioning Methods for Rapid Collection of 3-D Microstructure Data," JOM, 63 (3) (2011), 25-29.

18. Q. Feng et al., "Femtosecond Laser Micromachining of a Single-Crystal Superalloy," Scripta Materialia, 53 (5) (2005), 511-516.

19. M.S. Titus et al., "Dislocation Injection in Strontium Titanate by Femtosecond Laser Pulses," Journal of Applied Physics, 118 (7) (2015).

20. M.P. Echlin et al., "A New TriBeam System for Three-Dimensional Multimodal Materials Analysis," Review of Scientific Instruments, 83 (2) (2012).

21. M.P. Echlin et al., "The TriBeam System: Femtosecond Laser Ablation in situ SEM," Materials Characterization, 100 (2015), 1-12.

22. D.D. Krueger, R.D. Kissinger, and R.G. Menzies. "Development and Introduction of a Damage Tolerant High Tempearture Nickel-Base Disk Alloy, René 88DT," Superalloys 1992, ed. S.D. Antolovich et al. (Warrendale, PA: The Minerals, Metals \& Materials Society, 1992), 277-286.

23. S.T. Wlodek, M. Kelly, and D.A. Alden. "The Structure of René 88DT," Superalloys 1996, ed. R.D. Kissinger et al. (Warrendale, PA: The Minerals, Metals \& Materials Society, 1996), 129-136.

24. M.A. Groeber and M. Jackson, "DREAM.3D: a Digital Representation Environment for the Analysis of Microstructure in 3D," Integrating Materials and Manufacturing Innovation, 3 (1) (2014), 1-17.

25. Z. Wu and J.M. Sullivan, "Multiple Material Marching Cubes Algorithm," International Journal for Numerical Methods in Engineering, 58 (2) (2003), 189-207.

26. D.A. Field, "Laplacian Smoothing and Delaunay Triangulations," Communications in Applied Numerical Methods, 4 (6) (1988), 709-712.

27. S. Patala, J.K. Mason, and C.A. Schuh, "Improved Representations of Misorientation Information for Grain Boundary Science and Engineering," Progress in Materials Science, 57 (8) (2012), 1383-1425.

28. S.I. Wright and M.M. Nowell, "EBSD Image Quality Mapping," Microscopy and Microanalysis, 12 (2006), 72-84. 